method has a similar effect on man under these circumstances. In some instances, moreover, this dormant power of the specific resistance may be awakened by sensitised vaccine even at a comparatively late stage in the attack. In cases in which the treatment did no good there was no evidence to show that it dill the slightest harm.

The method shnuld have a great future prophylactically in the face of epidemics. It seems possible that sensitised vaccine may also be useful prophylactically in hospitalse.g., by this means the resistance of the patient might be raised to bacillus coli, streptococcus pyogenes, or the pneumococcus before operations on the alimentary tract or other infected area. Similarly, the method is open to the physician for the purpose of preventing secondary infections. Further, a prophylactic dose of sensitised vaccine might be given in cases of difficult labour.

Finally, I submit that in cases already infected the evidecce here brought forward goes to show that in a proportion of instances it is possible by this method to materially promote the patients' recovery. By administering a sensitised vaccine to these patients, we appear to bring into action available reserves in that complex and still incompletely defined entity, the patient's specific resistance.

Biblingraphy.-Besredka: Comptes Rendus de l'Academie des Sciences. Paris, vol. exxxiv., p. 1330. Bulletin de l'Institut Pasteur, Paris, 1910, pp. 241-53; Biologica, Revue Scientifique de Médecine, Dec. 15th, 1912. Broughton-Alcock : Comptes Rendus de l'Acrdémie des Suiences, Paris, 1912 . vol. cliv., p. 1523 ; THE LA NoET, April 26th, 1913, p. 1155. Levg and Aoki : Zeitschrift für Immunitätsforschung Jena. 1910, Band vii., p. 435, Orig. Gordon: Quarterly Journal of Medicine, July, 1912

Harley-street, $W$.

\section{A PRACTICAL METHOD OF GROWING THE ACNE BACILLUS FROM THE COMEDO FOR THE PREPARATION OF VACCINES.}

BY T. H. C. BENIANS, M.R.C.S. ENG., L.R.C.P. LoND., JUNIOR ASSISTANT, INOCULATION DEPARTMENT, LONDON HOSPITAT.

\section{(From the Bacteriological Department, London Fospital Medical College, Professor W. Bulloch, F.R.S., Director.)}

No attempt is made to enter at length into the controversial topic of the etiology of the disease. The relationship of the staphylococcus and acne bacillus as causative agents of the various lesions coming under the head of acne vulgaris is still to some extent unsettled. In passing, however, the writer gives it as his opinion that, judging on present evidence, the acne bacillus is the agent chiefly concerned in the immediate production of the skin lesions. Among these very protean lesions some are from their anatomical position completely beyond the scspe of vaccine therapy; in others the conditions are ideal for attaining the best-results by this method of treatment.

It is taken that the position of vaccines in the treatment of this disease, although not entirely satisfactory, is yet sufficiently well eitablished; the question now remains as to the relative values of autogenous as compared with stock vaccines. As a general rule, at any rate in hospital practice, it is customary to use a stock vaccine, so that this question has probably not yet been sufficiently well tested. In staphylococcal lesions we know that occasionally an autogenous vaccine may effect a cure where a stock vaccine has failed, and this although we are unable to differentiate varied strains of staphylococcus aureus. In the case of the acne lesions we have some evidence already that the organisms found in the lesions belong to a large and varied group of bacilli.

In a number of cases under treatment by the author the patients were changed from treatment with a stock acne vaccine to an autogrenous vaccine with very beneficial results; in some cases a complete cure of the pustular lesions was quickly effected. Data by other workers on this subject are, however, needed, because no more direct proof than a general consensus of opinion is possible. It may well be that some, or many, of the numerous failures in the treatment of acne by vaccines are to be attributed to the use of a stock vaccine, which vaccine may have been prepared from an organism totally differ $n$ t in its characters from that growing on and in the patient's skin.
Although the cultural characteristics of the acne bacillus are now fairly well known, the preparation of an autogenous vaccine for a patient remains still a matter of considerable difficulty. The principal methods of isolation and culture so far published are those described by Fleming ${ }^{1}$ and by Südmerson and Thompson. ${ }^{2}$ Fleming advocated the use of an oleic acid agar; he obtained the material from the smaller pustnles, and grew it in the first instance anaerobically. The latternamed writers use an acid serum agar; they take the deeper parts of the comedo in which the acne bacillus usually predominates, emulsify this in saline, and spread tbinly on the slope, so that they may obtain isolated colonies to pick off. They also made the first cultures anaerobically. Most workers, however, agree that to enable the acne bacillus to start growing on artificial media a considerable mass of the infective matesial must be planted, even on a medium which is favourable to the growth of the organism. When this is done it mostly happens that there are sufficient staphylococei present to overgrow the more slowly growing acne bacilius.

The method used by the writer does not aim at producing the organism in a state of bacteriological purity. The aim of the procedure is to supply in a reasonably short time a sufficiently free growth of the acne bacillus for the purpose of preparing a vaccine. In the more complete methods, moreover, the bacteriologist must himself remove the pus, or the comedo, from the patient, and at once proceed to select the required parts of it and to plant them out under anaerobic conditions. By the simple method here described the bacteriologist requires merely one or two comedones, whether expressed a few minutes or days before is immaterial.

As will be seen, a very small proportion of staphylococci are almost invariably present, but these may be treated as a negligible factor. The proportion of staphylococci present is not more than about 10 per cent. at the most ; thus in giving an average dose of the acne vaccine, say, 5,000,000, one is giving at the same time 500,000 staphylococci. Since an average dose of the staphylococci, perhaps to be given at the same time with the acne. is not likely to be less than $250,000,000$, the original 500,000 contained in the acne vaccine may well be overlooked.

Variations in the bacteriolngy of the comedo. - The bacteriology of the comedo varies greatly from a quantitative point of view. Almost always both staphylococci and acne bacilli are present. The staphylococcus may be said to be invariably present; the acne bacillus is sometimes, though rarely, absent. The average sebaceous plug contains acne bacilli greatly in excess of the staphylococci, which latter are mainly present in the superticial part. If such a comedo be put into neutral broth and incubated for about a week a large excess of staphylococcus will be present; after two weeks or more the numbers of cocci and bacilli will be more or less in equal proportion; in the course of a further week or two the bacilli may come to outnumber the cocci, although the amount of growth will not in any case be very great. If, on the other hand, the comedo starts with a relatively large proportion of staphglococci present, it is probable that under the conditions just mentioned the acne bacillus will make practically no growth at all. By the method to be described, it is rarely that one fails to obtain a considerable growth of the acne bacillus within a period of from 10 to 14 days. In the case of a comedo containing a large excess of bacilli, a culture practically pure and sufficiently abundant for the preparation of a vaccine may of ten be obtained by the fifth or sixth day.

In the preparation of some 20 autogenous vaccines by the author's method he has only failed once to obtain a satisfactory growth of the acne bacillus. On this occasion a large at ypical staphylococcus apparently was able to adapt its - lf to the altered cultural conditions; it started to grow on about the sixth day. and in the course of another week it bad completely overgrown the acne bacillus. Subcultured on to asar this organism failed to grow under either aerobic or anaerobic conditions.

The method of culture.-The culture method consists merely in growing the organism in a tube of neutral broth, the surface of which has been covered with about half an inch of sterile olive oil or sterili-ed ordinarv lard In iartice a

I THE Lancer, April 10th, 1909, p. 133.

2 Journal of Pathology and Bacteriology, vol. xiv., 1909, p. 224. c 02 
comedo is expressed into a sterile glass-tube of small bore, the edges of which have been previously well rounded in the flame. The comedo is dropped into the broth and the oil or melted lard added with a pipette. The comedo should be made to sink to the bottom, otherwise the growth may cling to the oil on the surface of the broth. After 24 or 48 hours a slight turbidity of the broth is noticeable, and this is due to the presence of staphylococcus albus; the growth of this organism, however, does not proceed any further. After three or four days' incubation the acne bacillus growth begins to show as a granular deposit at the bottom and sides of the tube, or sometimes in the form of small granules clinging to the oil. Films of the deposit made at this time usually show a mixture of about equal numbers of cocci and bacilli. At the end of a week or ten days' growth the number of cocci is relatively quite insignificant. Films of the deposit now made show a few isolated and scattered cocci and large masses of bacilli mostly of a uniform size. Often there is a great predominance of short oval or coccoid forms; these are readily differentiated from the staphylococcus by their shape and disposition. The bacilli clinging to the oil are generally larger and thicker than those in the deposit.

Preparation of vaccine.-The vaccine can be made up usually on the eighth or tenth day. The deposit is best removed with a Pasteur pipette ; it readily forms a uniform emulsion when mixed with saline and shaken vigorously in a bulb for a short time. The advantage or otherwise of the presence of a certain amount of the culture medium in bacterial vaccines is a debatable point, and is now being investigated. For the present, the writer advises that the bacilli should be thrown down in the centrifuge free from the broth medium; this supernatant fluid is then pipetted off and the bacteria re-emulsified in saline.

In explaining the rationale of this method of culture we can to some extent compare it with the conditions obtaining in the diseased sebaceous glands, which conditions it was designed to imitate. In the infected gland we find the outlet plugged with a mass of solid matter. The comedo filling the duct cavity may be solid throughout, but sometimes the deeper parts are semi-fluid in consistency. The bacilli lie, as it were, in a tube formed by the basement membrane of the gland, and are nourished presumably by the diffusing lymph and body fluids which pass through the membrane, on the one hand, and by the fatty substances derived from the degenerating epithelial cells on the other. The conditions here must, moreover, be chiefly anaerobic.

In our attempt to culture a comedo or other material containing a mixture of staphylococci and acne bacilli in a fluid medium we have to remember that the characteristics of these organisms are diametrically opposed. The staphylococcus in culture grows readily and rapidly; the acne bacillus commences its growth in artificial media with difficulty, and grows relatively slowly. We have, therefore, at once to discourage the growth of the staphylococcus and encourage the acne bacillus. Anaerobic conditions keep down the growth of the staphylococcus, and, to some extent, help the growth of the acne bacillus. Both organisms grow very freely in the presence of fat provided other conditions are favourable.

If a layer of fat or oil could be regarded as quite impermeable to oxygen, we should clearly have the ideal conditions required to inhibit the staphylococcus and assist the growth of the acne bacillus. The explanation, however, is not quite so simple as this, since oil is known to be permeable to oxygen. The true explanation probably is that the alkali formed by the staphylococci in the early stages of growth combines with the under surface of the oil and forms here a layer of soap which completes the anaerobic barrier. If anaerobic conditions alone be brought into use, as by covering the broth over with liquid paraffin, or by placing the culture in a Büchner's tube, a moderately pure growth of the acne bacillus may sometimes be obtained; it is, however, much less in amount than when fat is present in the tube.

SOUTH LONDON HOSPITAL FOR WOMEN (Incorporated), South Side, Clapham Common.H.R.H. Princess Louise, Duchess of Argyll, will lay the foundation stone of the hospital on Tuesday, July 1st, at 4.30 P.M.

\section{Clinital aldotes:}

MEDICAL, SURGICAL, OBSTETRICAL, AND THERAPEUTICAL.

\section{THE TREATMENT OF PERSISTENT DIPHTHERIA INFECTION BY MEANS OF DIPHTHERIA} ENDOTOXIN.

By R. Tanner Hewlett, M.D. Lond., F.R.C.P. Lond., PROFESSOR OF BACTERIOLOGY IN THE UYIYERSITY OF LOYDON.

Is a former paper ${ }^{1}$ Dr. A. T. Nankivell and I detailed results obtained in the "treatment" of persistent diphtheria infection by means of one or more injections of diphtheria endotoxin prepared by the Macfadyen method. 'There were 13 cases in which diphtheria bacilli had persisted in the throat or nose or both for weeks or months after an attack of diphtheria, and in which the bacilli disappeared aftex 1-3 doses of the diphtheria endotoxin. There were 5 other cases in which the bacilli did not disappear with the use of endotoxin, but the doses of endotoxin given were much smaller than in the successful series. We were inclined to believe that the use of the endotoxin is a promising means of treating these troublesome cases.

Another case of persistent diphtheria infection after an attack of diphtheria has recently been treated by me with diphtheria endotoxin with subsequent disappearance of the diphtheria bacilli. The endotoxin was the same preparation as that previously employed, and was about $2 \frac{1}{2}$ years old. Judging by the local reaction produced, the endotoxin, kept in an ordinary cupboard, has maintained its properties but little impaired by this long keeping. The following are the details of the case.

A young medical man had a mild attack of diphtheria at the end of January, 1913. Jan. 28th: Sore throat with patch on left tonsil. Jan. 29th: Swab taken from patch. Jan. 30th : Diphtheria bacilli found in culture; 6000 units of diphtheria antitoxin injected. The patch cleared from the tonsil within 12 hours. Examinatiuns for diphtheria bacilli were then made at intervals and diphtheria bacilli were found to persist. Finally, two injections of endotoxin were given. The following table shows the results of the cultures:-

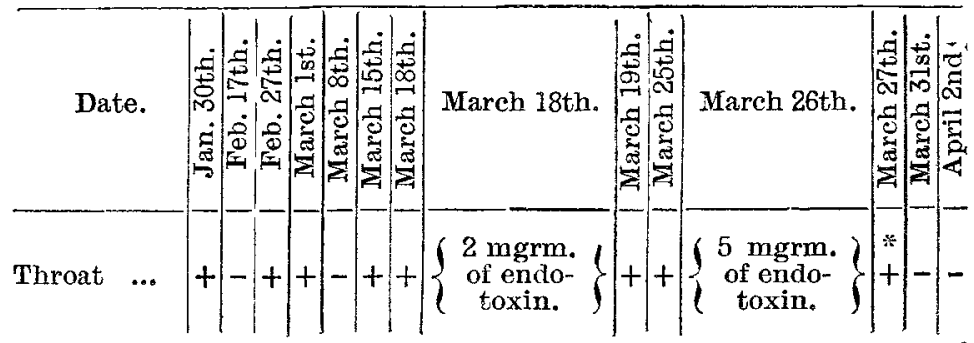

+ sign indicates "diphtheria bacilli found," and - sign "diphtheria bacilli not found." * Very few bacilli found after much searching (March 27th).

The injections of the endotoxin caused no general disturbance, but redness, swelling, tenderness, and aching were present at the seat of injection, which was into the triceps region of the arm.

The successful result in this case may or may not have been due to the treatment with endotoxin, but it certainly confirms the view previously taken by Dr. Nankivell and myself that the endotoxin treatment is worthy of trial in persistent diphtheria infections.

Chandos-street, W.C.

\section{A CLINICAL TEST FOR MALARIAL FEVER.}

Bх J. M. Atkinson, M.B. Lond, M.R.O.S. EnG., D.P.H. САMB.,

TATE PRINCTPAL CIVIL MEDICAT, OFFICER, HONG-KONG.

AT the Second Biennial Congress of the Far Eastern Association of Tropical Medicine held in Hong-Kong last year, Dr. Justi drew attention to the fact that Schlesinger's reaction for demonstrating the presence of urobilin in the

1 The Treatment of Diphtheria Infection by means of Diphtheria Fndotoxin, THE L A Y YCET, July 20th, 1912, n. 143 . 